\title{
Engineering ultra long charge carrier lifetimes in organic electronic devices at room temperature.
}

Yajun Gao (高亚军), ${ }^{1,2, * *}$ Roderick.C.I. Mackenzie ${ }^{3, *, * *}$, Yang Liu (刘洋) ${ }^{1}$, Bin Xu (徐斌) ${ }^{1}$, Paul. H. M. van Loosdrecht, ${ }^{1,2,4 \$}$ and Wenjing Tian（田文晶） $1, \#$

${ }^{1}$ State Key Laboratory of Supramolecular Structures and Materials, Jilin University, 2699 Qianjin Street, Changchun 130012, China.

${ }^{2}$ Optical Condensed Matter Physics, Zernike Institute for Advanced Materials, University of Groningen, Nijenborgh 4, 9747AG Groningen, The Netherlands.

${ }^{3}$ Faculty of Engineering, The University of Nottingham, University Park, Nottingham, NG7 2RD, UK

${ }^{4}$ II. Physikalisches Institut, University of Cologne, Zülpicher Strasse 77, 50937 Cologne, Germany.

Corresponding authors:

*roderick.mackenzie@ nottingham.ac.uk

\$pv1@ph2.uni-koeln.nl

\#wjtian@jlu.edu.cn

**Both authors contributed equally to this work.

\begin{abstract}
Herein, we report on the observation of a one hour long charge carrier lifetime in a plastic electronic device at room temperature, this charge carrier lifetime figure is over four orders of magnitude longer than previously reported. We attribute this to electrons and holes being spatially separated shortly after photogeneration by the potential gradients distribution set up within the device by doping. Numerical modeling suggests that the band structure of the device resembles a potential hill, which forces photogenerated electrons to drift towards the contacts, while trapping photogenerated holes in an electron poor region in the center of the device. Thus we demonstrate that by carefully engineering the potential profile in organic semiconductor devices the carrier lifetime can be tuned over nine orders of magnitude in time. Devices with ultra long carrier lifetimes open up the possibility for new classes of plastic electronic devices such as ultra low light level photodetectors.
\end{abstract}




\section{Introduction}

Plastic electronic devices fabricated from carbon based molecules and polymers [1] have received considerable academic and industrial attention over the last decade [2]. They offer the promise of flexible and low cost broad area devices, which can be fabricated using high speed, low temperature roll-to-roll production techniques [3]. Devices such as organic LED (OLED) displays/lighting [4,6], organic photovoltaic (OPV) devices [5], organic field effect transistors (OEFTs) [7], and photodetectors [8-11] have been demonstrated. However, organic semiconductor materials are inherently more disordered than their inorganic counterparts [12], due to a lack of a regular crystalline lattice. Thus charge carrier recombination rates are generally higher, while mobilities are generally around three to four orders of magnitude lower [13]. This means that, although organic devices offer the potential for lower cost devices, performance is in general not as good as their inorganic counterparts.

Recently there has been considerable attention focused on understanding the relationship between mobility [13], recombination [14] and the distribution of carrier transport states within organic semiconductors [15] with the objective of improving carrier lifetime/transport and thus device performance. Methods such as transient photo-voltage [16], photo charge extraction by linearly increasing voltage (photo-CELIV) [17] and impedance spectroscopy [18] have been applied to obtain mobility and recombination data. Charge carrier lifetimes in the range of $1 \mathrm{ps}-0.3 \mathrm{~s}$ have been reported from devices fabricated from both solution processed polymers [21,22,23] and evaporated small molecules [24-28]. These measurements have in general been performed on working devices where the band edge of the metallic contacts (typically aluminum and ITO), has been aligned to the HOMO/LUMO level of the semiconductor to increase carrier injection and extraction efficiency. However, the equilibrium Fermi level of the organic semiconductor is usually mid way between the HOMO - LUMO level, and far from that of the ITO or Aluminum contact. This means for the system to reach equilibrium, and for the quasi-Fermi levels of the metal and semiconductor to align, a significant number of both electrons and holes must be injected from the contacts into the bulk of the organic semiconductor, resulting in a large equilibrium background carrier population. The nongeminate recombination rate $(\mathrm{R})$ in organic semiconductors is known to be a strong function of both local electron $(\mathrm{n}(\mathrm{x}))$ and local hole carrier $(\mathrm{p}(\mathrm{x}))$ densities [20], $R(x)=k(x, n, p) n(x) p(x)$ [29], where $\mathrm{k}$ is a constant of proportionality and $\mathrm{x}$ is the position. Thus if the local background charge density of either species could be reduced, so would the recombination rate. Furthermore, if photogenerated electrons could be quickly swept from hole rich regions, and photogenerated holes 
from electron rich regions, the spatial overlap of the photogenerated populations would be reduced and charge carrier lifetime would also be increased.

In the following pages we demonstrate that by engineering the band structure in a plastic electronic device, hole carrier lifetimes longer than one hour can be achieved. We attribute this four order of magnitude increase in carrier lifetime to a hill shaped band structure which forces photogenerated electrons and holes to spatially separate shortly after generation, the reduction in spatial overlap between the electron and hole population reduces the recombination rate. The hill shaped band structure also generates a hole rich and electron poor region in the center of the device in which the hole recombination rate is low, this acts as a charge storage area. These results demonstrate that it is possible for organic semiconductors to have long charge carrier lifetimes, and opens up the possibility for new classes of ultra low light level photodetectors and memory elements.

\section{Experimental}

Figure 1a depicts the device structure; it consists of an ITO coated glass substrate with contact resistance $30 \mathrm{Ohms} / \mathrm{square}$. An insulating layer of $\mathrm{SiO}_{2}$ is spin coated from a sol-gel onto the ITO film, followed by a layer of the organic semiconductor N,N'-Di-[(1-naphthyl)-N,N'-diphenyl]-1,1'biphenyl)-4,4'-diamine (NPB). Finally a $30 \mathrm{~nm}$ thick aluminum capping electrode is evaporated onto the NPB. Full details of device fabrication can be found in the supplementary information. Charge is stored within the device by photoexcitation through the transparent ITO contact using a $355 \mathrm{~nm}$ laser with a $10 \mathrm{~ns}$ pulse length and a fluorescence of $110 \mu \mathrm{Jcm}^{-2}$. The flat band structure of the device is depicted in figure $1 \mathrm{~b}$ and the chemical structure of NPB is shown in figure 1c. To measure the charge stored within the device, a positive voltage ramp of duration $t_{\text {extract }}$ and magnitude $\mathrm{V}_{\max }$ is applied to the ITO contact. This voltage ramp is applied at a time $\mathrm{t}_{\text {delay }}$, after the optical excitation ends. The timings of the voltage transients are shown in figure $2 \mathrm{a}$.

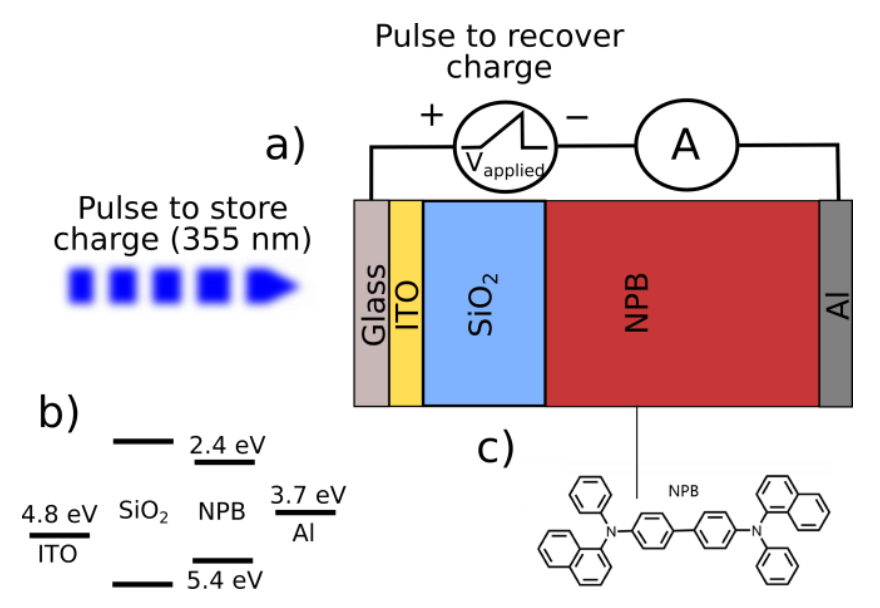

Figure 1: a) The Glass/ITO/SiO2/NPB/Al structure of the device, b) The flat-band energy diagram of the device. c) The 
chemical structure of NPB. Also visible in the figure are the $355 \mathrm{~nm}$ laser excitation used to store charge in the device, the voltage source used to extract charge from the device and the ammeter used to count the extracted charge. It should be noted that because the SiO2 layer is deposited from solution it is not a perfect insulator. (see SI for more information)

Two typical current transients measured form the system are shown in Figure $2 \mathrm{~b}$ (black and red lines) along with the corresponding voltage ramp applied to the device (blue line). The black line represents the current measured from the device when no laser excitation is applied, while the red line shows the current transient when the device is photoexcited $1 \mathrm{~ms}$ before the start of the voltage ramp. In both cases it can be seen that when the applied voltage starts increasing at time $=0$, the current produced by the device rapidly increases reaching a plateau of $0.014 \mathrm{~mA}$ within $10 \mu \mathrm{s}$. This plateau of current corresponds to the geometric capacitive current being drawn out of the device from the contacts. Note, charge density was calculated by dividing the measured charge from the device by the volume of the NPB active layer. When no photoexcitation is applied to the device (black line), the current remains stable until $400 \mu$ s when the voltage ramp ends. For the case where the device is photoexcited (red line), a Gaussian shaped bump of charge appears on the transient starting at $50 \mu \mathrm{s}$ and ending by $200 \mu \mathrm{s}$. It has a maximum height of $0.03 \mathrm{~mA}$ at $90 \mu \mathrm{s}$. The area under this Gaussian of charge corresponds to the charge photogenerated within the device. It should also be noted that a positive bias is applied to the ITO contact, meaning that the extracted current is due to holes.
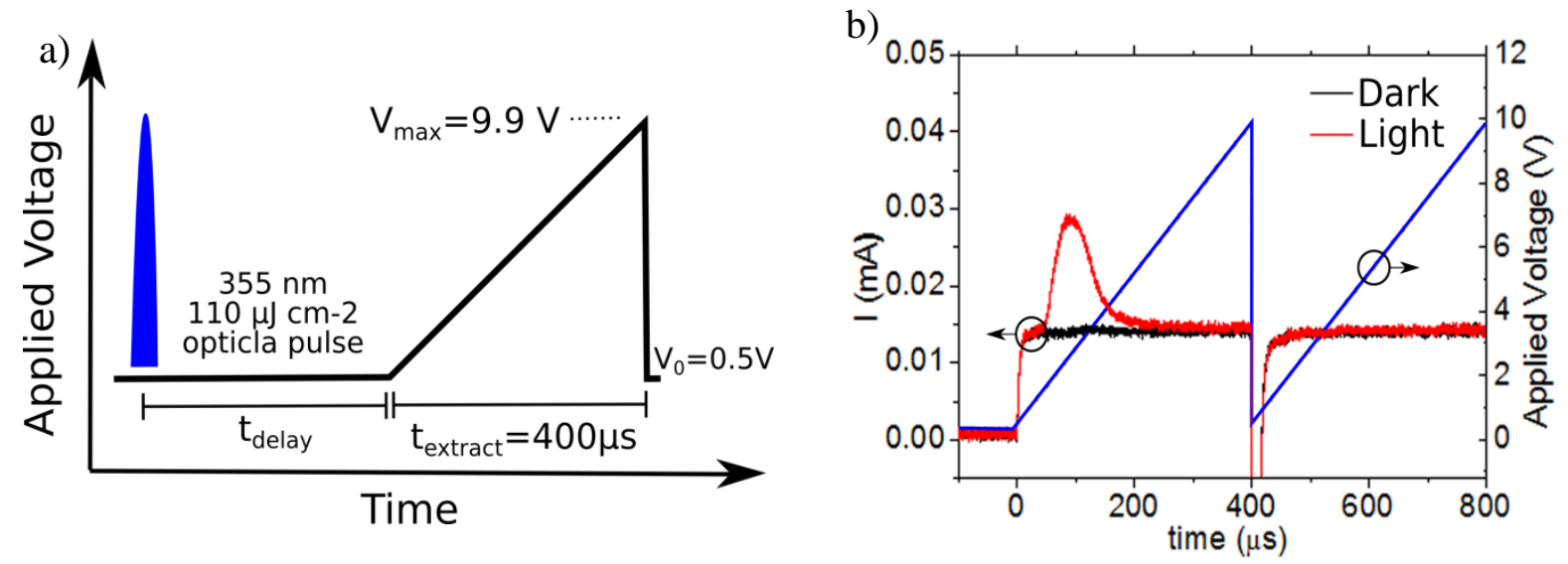

Figure 2:a) A schematic diagram of the voltage transient applied to the device to extract the photogenerated charge, the voltage transient ramps from Vo to Vmax. The figure also shows the optical excitation applied at tdelay before the voltage transient begins. b) Two typical current transients from the device when the device is photo excited (red line) and not photoexcited (black line); a time of $t_{\text {delay }}=1 \mathrm{~ms}$ was used in this measurement. 
Figure 3a plots current transients as a function of delay time between photoexcitation and extraction $\left(t_{\text {delay }}\right)$. The black line represents charge extracted from the device after $100 \mu$ s, while the blue line represents charge extracted from the device after one hour. Figure $3 b$ plots the integrated photogenerated charge from figure $3 \mathrm{a}$ as a function of time, it can be seen that after 1 hour around $25 \%$ of the photogenerated charge still remains in the device, suggesting that electron-hole recombination rate is much slower than previously observed in organic electronic devices.

Figure 3b-inset plots the photogenerated charge extracted from a device when the $\mathrm{SiO}_{2}$ carrier blocking layer is replaced with a polystyrene blocking layer. It can be seen that this device has a much higher recombination rate than the device with the $\mathrm{SiO}_{2}$ blocking layer, indeed the charge density decreases by an order of magnitude over the first $100 \mathrm{~ms}$. To investigate if the ultra long charge carrier lifetimes in the $\mathrm{SiO} 2$ sample are due to an interface effect or a bulk effect, $\mathrm{SiO} 2 / \mathrm{NPB}$ devices were fabricated with a thin PS layer separating $\mathrm{SiO}_{2}$ from the NPB. When the PS layer was thin $(19 \mathrm{~nm})$ long charge carrier life times were still observed, however when the thickness of the PS layer was increased charge carrier time decreased - see figure 3c. The ability to obtain long charge carrier lifetimes with a thin PS layer rules out interface trapping as the charge storage mechanism. Under these experimental conditions, $\mathrm{SiO}_{2}$ and PS are more or less identical in terms of their ability to electrically insulate. However, one key difference between these two materials is that $\mathrm{SiO}_{2}$ has an electrical permittivity of 3.9, while PS has a much smaller electrical permittivity of around 2.5. This suggests that distribution of electrostatic potential within the bulk of the device is key to determining long charge carrier lifetimes. To further support this assertion, we fabricated devices with an insulating layer consisting purely of Poly(methyl methacrylate) (PMMA). PMMA has a relatively high permittivity of $\sim 4.9$, and so if the distribution of electrostatic potential within the bulk of the device is important, one would also expect to also see long lived charge carrier lifetimes in this device. This was in fact the case, and we were able to extract charge tens of minutes after photoexcitation - see the SI for details.
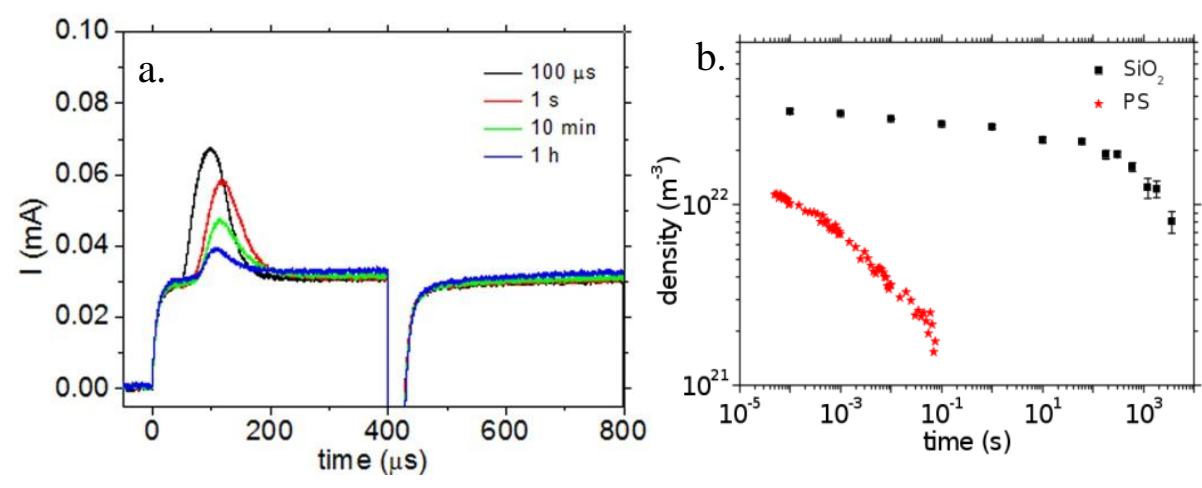

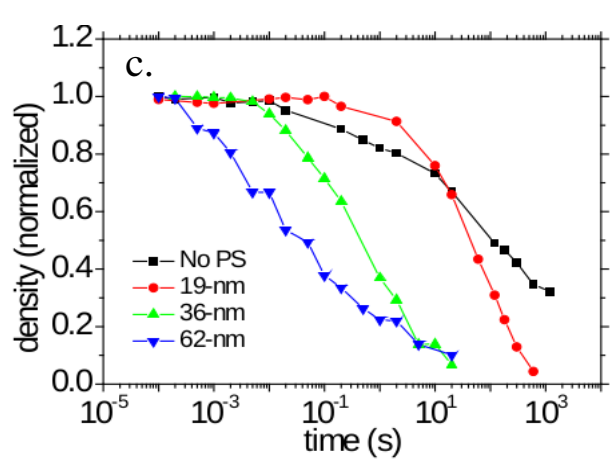


Figure 3: a) Charge extraction transients with varying times between photoexcitation and charge extraction ( $t$ delay). The current features after $400 \mathrm{~ms}$ is discussed in the SI. b) Black squares: integrated photocharge extracted from the device as a function of $t_{\text {delay }}$ corresponding to figure 3 a, note that it takes over an hour for the charge density to decay to $25 \%$ of its initial value. Red stars: Integrated charge from the device when the $\mathrm{SiO}_{2}$ blocking layer is replaced with a polystyrene blocking layer. By changing the blocking layer the carrier lifetime is significantly reduced. c) Charge carrier lifetimes for a ITO/SiO2/PS/NPB/Al device where the thickness of the PS layer is varied.

\section{Discussion}

Previously carrier lifetimes in organic systems have been observed in the range $1 \mathrm{ps}-0.3 \mathrm{~s}$ $[17,18,19]$, thus observing carriers with a lifetime more than four orders of magnitude more than previously reported was not expected. If the current transient obtained after photoexcitation in figure $2 \mathrm{~b}$ is examined (red line) it can be seen that until around $50 \mu$ s after the voltage ramp has been applied no photogenerated charge is extracted. Only once the applied voltage has reached the value of $1.68 \mathrm{~V}($ at $50 \mu \mathrm{s}$ ) can the photogenerated charge be accessed. The need for an applied bias of over one volt to extract photogenerated charges, suggests that there is an energetic barrier between where the charge is stored in the device and the extracting contact.

The presence of an energetic barrier hindering charge extraction suggests the presence of a Schottky contact and associated band bending separating the stored photogenerated charge within the device from the extracting contact. Schottky contacts and band bending in electronic devices are associated to regions of space charge, which in turn is associated with material doping. Although the NPB layer is not intentionally doped it has been frequently reported that contaminants, such as water, oxygen or non pure materials can lead to unintentional and relatively high levels of background doping in organic devices $[31,26,27]$.

From figure $3 \mathrm{c}$ we can see that by changing the material used in the blocking layer from $\mathrm{SiO}_{2}$ to polystyrene the charge carrier lifetime can be significantly reduced. The choice and electrical permittivity of insulating material will dictate the exact distribution of potential within the device, how much charge is injected into the device from the contacts to establish equilibrium conditions, where the charge resides within the device, and consequently the recombination rate. When the aluminum capping contact is replaced by a copper contact, the same long carrier lifetime was observed, but the shape of the extracted photoexcited charge packet changed (Figure S3 in SI). This further suggests that the long lived charge carriers are not an intrinsic property of the material but rather a function of the carrier distribution with in the device. 
Finally, it should also be noted that the current transients measured from this device upon the application of a voltage ramp are very different in nature to those observed from organic solar cells, which are fabricated not to have an energetic barrier between the contacts and where charge is generated. Thus in organic solar cells there is no delay between the application of the voltage transient and extraction of photogenerated charge [30].

\section{Theory}

To better understand why the device can store charge for long periods of time with very little recombination occurring, and why an applied bias of over one volt is required to extract the stored charge, we model the device using a time domain drift-diffusion model. To calculate the electrostatic potential distribution between the ITO contact $(x=0)$ and the Al top contact $(x=d)$, Poisson's equation,

$$
\frac{d}{d x} \cdot \varepsilon_{0} \varepsilon_{r} \frac{d}{d x} \phi=q\left(n_{f}+n_{t}-p_{f}-p_{t}-N_{a d}\right)
$$

is solved over the $\mathrm{SiO}_{2}$ layer and the NPB semiconductor. Where, $\phi$ is the position dependent potential, $\varepsilon_{0}$ is the permittivity of free space, $q$ is the charge of an electron, $N_{a d}$ is the density of dopant molecules, and $n_{f} / p_{f}, n_{t} / p_{t}$ are the densities of free and trapped electrons/holes respectively. To account for drift and diffusion of charge carriers within the device, the bi-polar drift diffusion equations are solved over the same region,

$$
\begin{aligned}
& J_{n}=q \mu_{e} n_{f} \frac{\partial E_{\text {LUMO }}}{\partial x}+q D_{n} \frac{\partial n_{f}}{\partial x} \\
& J_{p}=q \mu_{h} p_{f} \frac{\partial E_{\text {HOMO }}}{\partial x}-q D_{p} \frac{\partial p_{f}}{\partial x}
\end{aligned}
$$

where, $J_{n} / J_{p}$ are the electron and hole current densities, $E_{\text {Lимо }}, E_{\text {номо }}$ are the energy levels of the LUMO and HOMO respectively, and $\mu_{e}, \mu_{h}$ are the electron and hole mobilities. Non-germinate recombination and carrier trapping are described using the Shockley-Read-Hall recombination model [32]. Exponential carrier trap states between the HOMO and LUMO mobility edges are assumed [33]. The transfer matrix method is used to calculate the photon distribution within the device. A full description of the device model can be found elsewhere [34,35]. It should also be 
noted that we assume charge is generated within NPB in accordance to the process described in [36].

The $\mathrm{SiO}_{2} / \mathrm{NPB}$ sandwich structure is modeled, by using two layers of semiconducting material. The insulating $\mathrm{SiO}_{2}$ layer is assumed to be transparent and given a low mobility of $1 \times 10^{-10} \mathrm{~m}^{2} \mathrm{~V}^{-1} \mathrm{~s}^{-1}$ to prevent conduction of charge. While the NPB layer is assumed to have an optical absorption coefficient of $1 \times 10^{7} \mathrm{~m}^{-1}$ as measured using UV-Vis spectroscopy (see SI) and a mobility of $1 \times 10^{-6}$ $\mathrm{m}^{2} \mathrm{~V}^{-1} \mathrm{~s}^{-1}$, a full list of simulation parameters can also be found in the SI. The interface between the sol-gen deposited $\mathrm{SiO}_{2}$ and NPB is assumed to have a $2 \mathrm{eV}$ step (discussed in the supplementary information).

Figure $4 \mathrm{a}$ plots the simulated band structure within the device when the doping within the NPB layer is increased from $0 \mathrm{~m}^{-3}$ (red line), to a doping level of $7 \times 10^{22} \mathrm{~m}^{-3}$. It can be seen that as the doping level is increased the bands bend upwards forming a potential hill. Figure $4 \mathrm{~b}$, plots the corresponding current transients as a function of doping level, the same colors have been used to denote doping density as in figure 4a. It can be seen that as the level of doping increases, the Gaussian of charge due to photoexcited carriers being extracted from the device becomes wider and more delayed in time. When the doping reaches a level of $7 \times 10^{22} \mathrm{~m}^{-3}$ the transients start to resemble those in figure $2 \mathrm{~b}$. A Mott-Schottky analysis at a frequency of $1 \mathrm{kHz}$ [31] on a ITO/NPB(335nm)/Al structure confirmed that the devices are doped at a density of $5.2 \times 10^{22} \mathrm{~m}^{-3}$, the identity of the dopants is discussed in the SI.
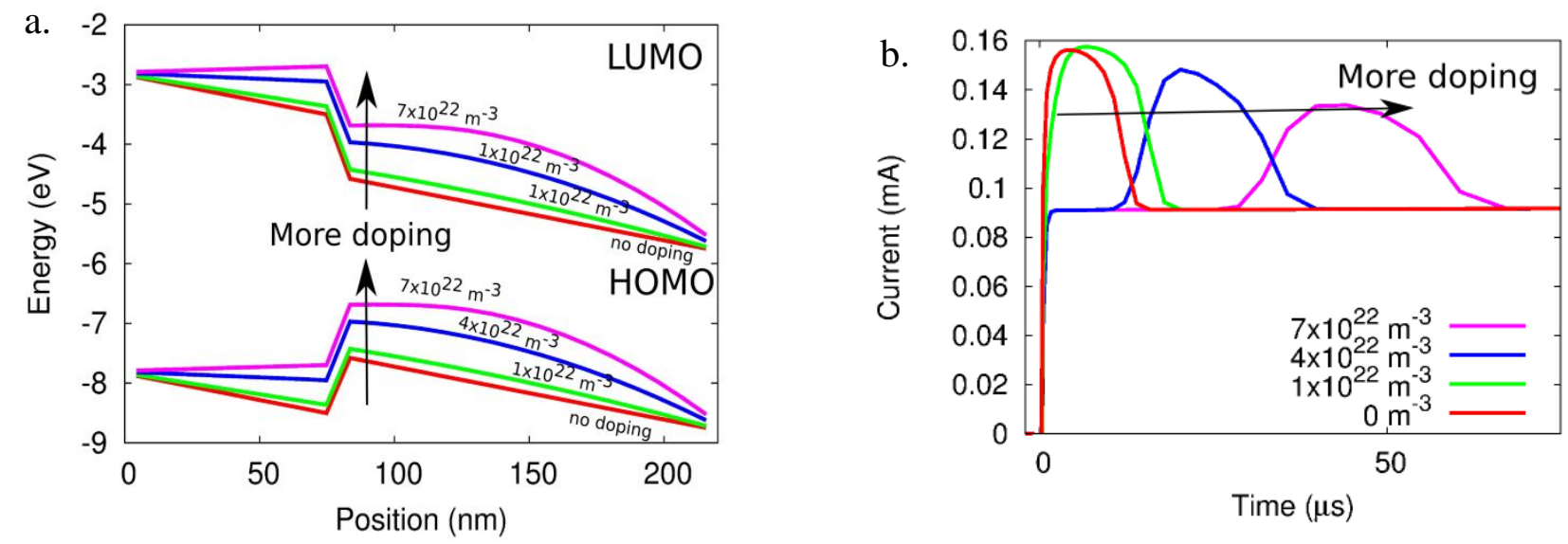

Figure 4:a) Band bending as a function of doping. As more doping is introduced the bands bend more. b) The corresponding current transients, it can be seen as doping increases the transients start to resemble more those of the experimental transients. The same line colors to denote doping have been used in both figures. The dopants are considered p-type because charge is extracted on application of a positive voltage. 
Figure 5 plots the proportion of photogenerated holes remaining in the device as a function of time after photoexcitation. The solid red line in figure 5 corresponds to the case when the device is undoped and hence there is no band bending (see the red line in figure 4a). As doping is increased it can be seen that it takes the carrier population longer to return to an equilibrium level after photoextitation - solid green line. When a doping level of $7 \times 10^{22} \mathrm{~m}^{-3}$ is used, no significant carrier recombination can be observed within the first $1000 \mu$ s. The curves denoted with points, represent simulations where the energetic step between the $\mathrm{SiO}_{2}$ and NPB has been removed. This represents the case where the $\mathrm{SiO} 2$ contains defects states allowing some charge to seep into it. It can be seen that a 'good' insulating layer is beneficial for long carrier lifetimes, high levels of doping are more important.

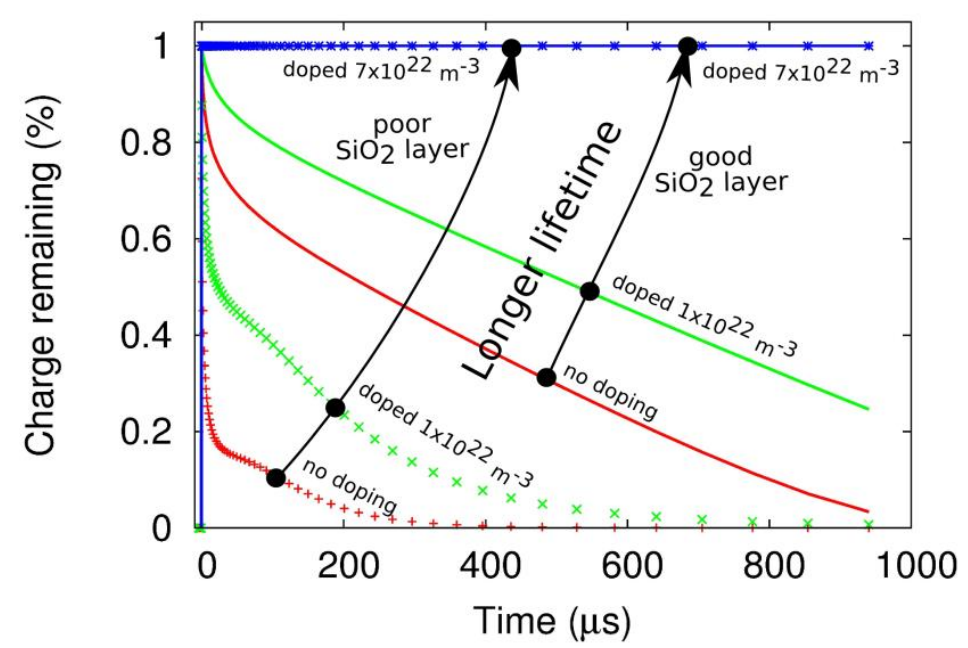

Figure 5: Photogenerated charge remaining in the device as a function of time. It can be seen that when the device is undoped (red line) almost all the photogenerated charge recombines quickly. As doping is increased (green line), carrier lifetime increases. When the doping level is further increased (blue line), no recombination can be observed within $1000 \mu$ s after photoexcitation. The dots represent the case where the energetic barrier between the SiO2 and $N P B$ is removed allowing charges to penetrate the SiO2, as could happen due to the presence of defect states.

The relationship between carrier lifetime and band bending can be understood by examining figure 6, where the electronic transport processes occurring within the first few microseconds before and after photoexcitation have been depicted. Figure 6a depicts the hill shaped band structure as seen in figure $4 \mathrm{a}$ (purple line) before photoexcitation. Figure $6 \mathrm{~b}$ plots the bandstructure of the device just after photoexcitation, photogenerated charge carriers are shown in green, as time progresses both electrons and holes attempt to minimize their energy. Thus the electrons drift down towards the contacts and become extracted (Indeed upon photoexcitation a negative current spike can be seen, representing the photogenerated electrons leaving the device - see SI for details). While the holes 
drift towards the center of the device and become trapped by the potential profile of the device and the $\mathrm{SiO} 2$ insulator layer. This process results in spatially separated electrons and holes. Because there is no spatial overlap between the electron and hole populations, charge carriers cannot recombine. Another important, feature of figure 6 (and 4a), is that the band structure is bent upwards. This places the equilibrium quasi-Fermi level (red dashed line in figure 6), near the HOMO band edge at the center of the device, meaning that the center of the device will be hole rich and electron poor. A low number of electrons in the center of the device will reduce the spatial overlap of the electron and hole populations and reduce hole recombination.

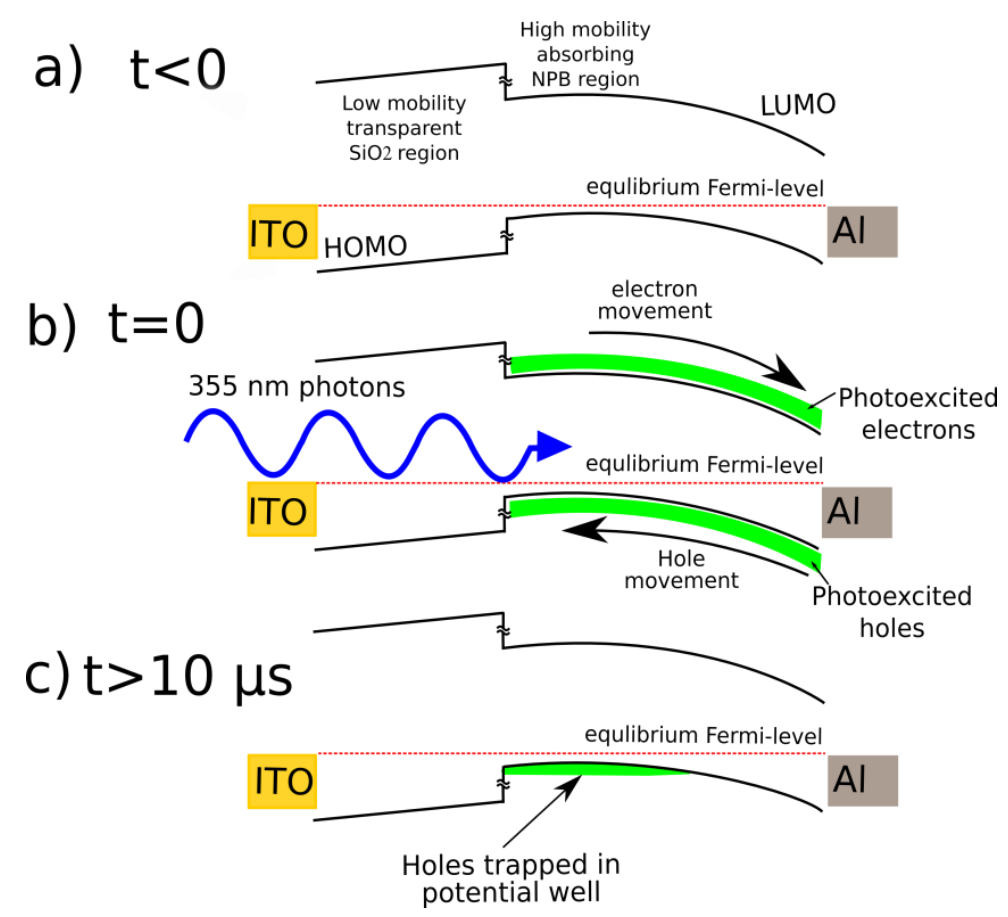

Figure 6: a) By introducing a small amount of doping into the device, the bands can be made to bend upwards. b) When the device is photoexcited charge is generated (green). c) As time progresses, due to the band bending, holes drift upwards in energy and get captured in the center of the device, whereas electrons move to the contact and become extracted. This produces spatial separation of electrons and holes and thus lowers the recombination rate. Another important feature of having upwardly bent bands is that in the center of the device where the photogenerated charge becomes trapped, the Fermi level is close to the HOMO, producing a hole rich-electron poor region, which will have a low hole recombination rate.

Figure $7 \mathrm{a}$ plots the simulated charge extraction process as the positive voltage ramp is applied to the left hand side of the device. The top panel plots the HOMO band structure as a function of increasing applied bias. It can be seen that as the applied potential is increased the highest point of the HOMO is pulled down, eventually reducing to the same height as the right hand extracting contact. Underneath the plot of the HOMO, the hole density as a function of position is plotted. It can be seen as the positive potential is applied to the device the photogenerated charge packet 
moves from the left hand side to the right, always trying to minimize its energy. At later times (not shown) the packet is fully extracted from the device. Figure $7 \mathrm{~b}$ summarizes the charge extraction process in pictorial form, the photogenerated charge can be seen being pushed from the left hand side of the device to the right.
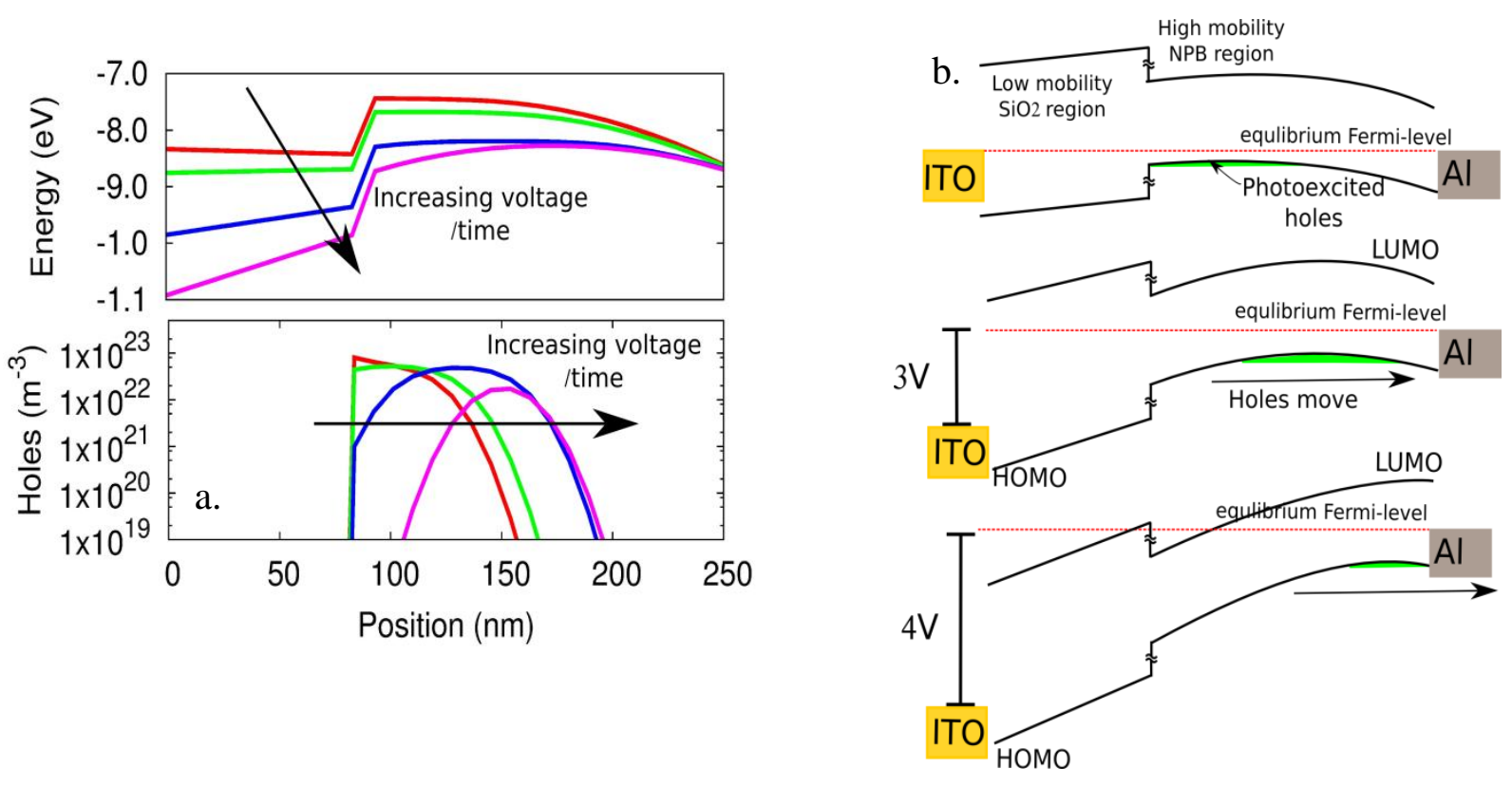

Figure 7: a) The top plot shows the simulated HOMO level as a function of applied bias, while the bottom plot shows the simulated charge packet moving to the right as the bias voltage is increased. $b$ ) A diagram summarizing the charge being pushed out of the device as a function of applied voltage as depicted in figure 7a. A short time after photoexcitation, holes sit in the center of the device. As a positive voltage is applied to the ITO contact, the charges move towards the extracting contact.

Above, we suggested that the difference in lifetimes observed between the $\mathrm{SiO}_{2}$ device and the PS device could be due to the different permittivities of the layers $\left(\varepsilon_{\mathrm{SiO} 2}=3.9\right.$ and $\left.\varepsilon_{\mathrm{PS}}=2.5\right)$. This observation can be better understood by examining figure 8a which plots simulated charge carrier lifetime as a function of carrier blocking layer permittivity. It can be seen from the figure that the higher the relative permittivity, the longer the lifetime of the charge carriers. This can be explained by examining figure $8 \mathrm{~b}$ where the band structure at equilibrium is plotted as a function of relative permittivity. The higher the relative permittivity of the insulating layer, the smaller the potential drop over the insulating layer and the further away the LUMO is from the equilibrium quasi-Fermi level. This will mean that for high permittivities, there will be few electrons and thus a low recombination rate in the region of the device where photogenerated charges are stored. 

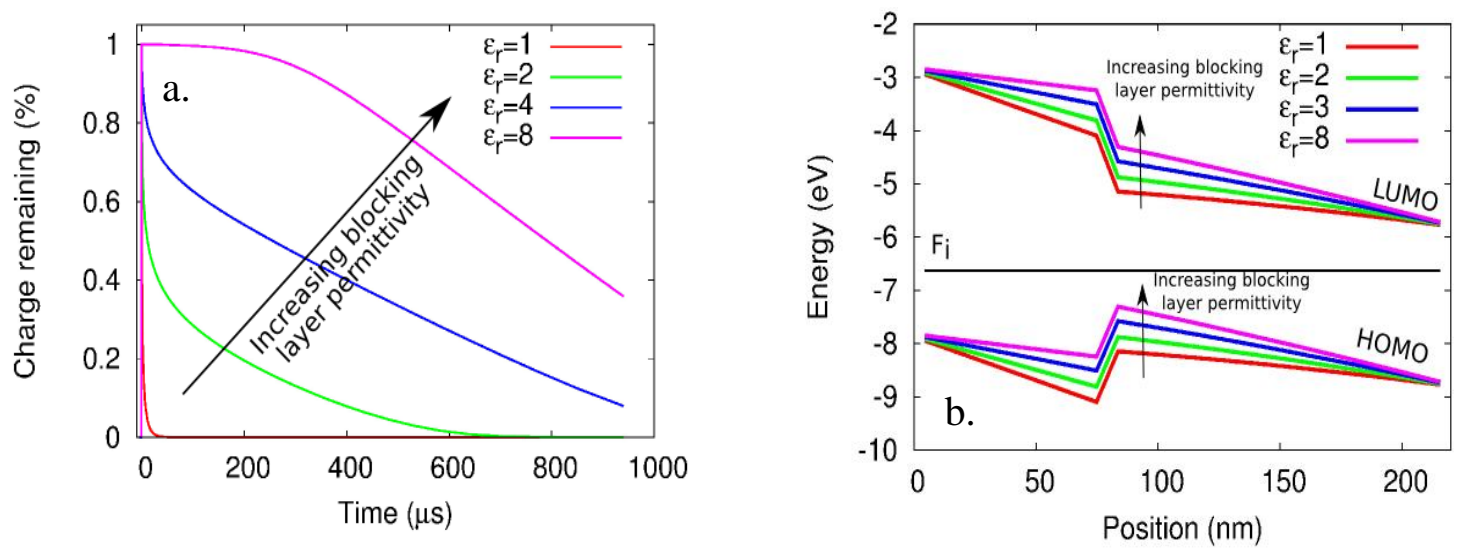

Figure 8a: Charge carrier lifetime as a function of carrier blocking layer permittivity. Figure 8b: The corresponding band structure.

Figure 9a plots the experimental current transients as a function of maximum applied voltage using a delay time of $10 \mu \mathrm{s}$. It can be seen that the as the maximum voltage $\left(\mathrm{V}_{\max }\right)$ is increased, the peak in the current due to the photoexcited charge shifts to the left and becomes narrower. The simulation of this process is depicted in figure $9 \mathrm{~b}$. That the simulation can predict these experimental trends, suggests that the model is a good approximation to the physical processes occurring within the device.
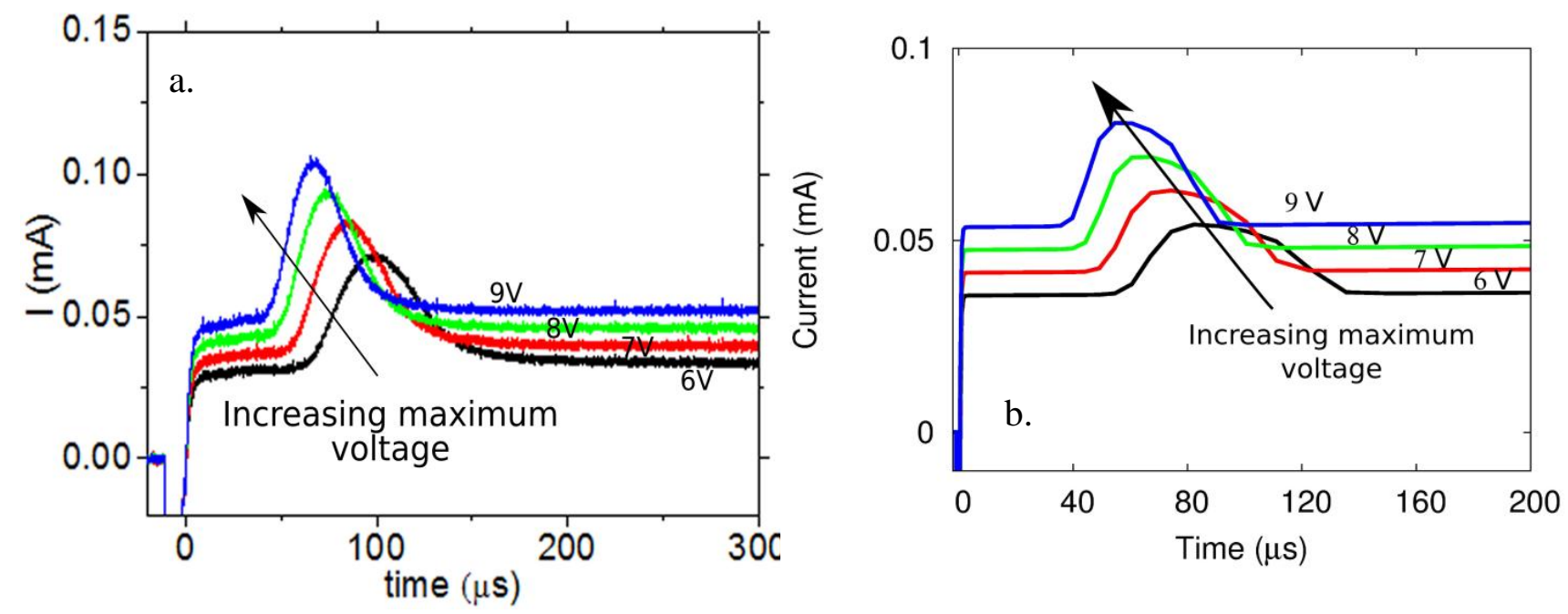

Figure 9: Experimental (a) and simulated (b) charge extraction transients as a function of $V_{\text {max }}$. If the experimental and simulated results are compared, it can be seen in both data sets, that as $V_{\max }$ is increased the current peak shifts to the left and narrows.

\section{Applications for long life time charge carriers}

Detecting ultra low power optical signals, such as light from distant stars, is important in science, medical, and defense applications. The ability of the structure to produce long-lived photogenerated 
charges, opens up the possibility to use the device in a time-intergrating mode, whereby a very low photon flux over long periods of time cause the gradual build up of charge. Once enough charge carriers have been collected to make the signal detectable by the external electronics, charge could be extracted. To demonstrate this, a ITO/SiO2(105 nm)/NPB(70 nm)/Al structure was constantly illuminated at $365 \mathrm{~nm}$ using a low brightness source over exposure times ranging from 1s to 7000s. At the end of the measurement the voltage ramp was applied to the device to extract the charge. The experiment was performed at light fluxes of 1.6, 3.2, 4.8 and $6.4, \mu \mathrm{W} \mathrm{cm}{ }^{-2}$. The results are plotted in figure 10. It can be seen that, as expected, a gradual buildup of charge is observed over time.

Another potential use of ultra long lived charge carriers could be in optically writable electronically readable memory elements. Charge could be stored in the device with a light pulse and the device's state measured or reset by applying the voltage ramp. In figure $3 \mathrm{~b}$ we demonstrated that charge can be stored in this device for more than 1 hour, however it should be noted that we expect longer lifetime to be accessible with a higher quality $\mathrm{SiO}_{2}$ layer, controlled doping of the semiconductor layer, and possibly by using an insulating layer with a higher permittivity than $\mathrm{SiO}_{2}$.

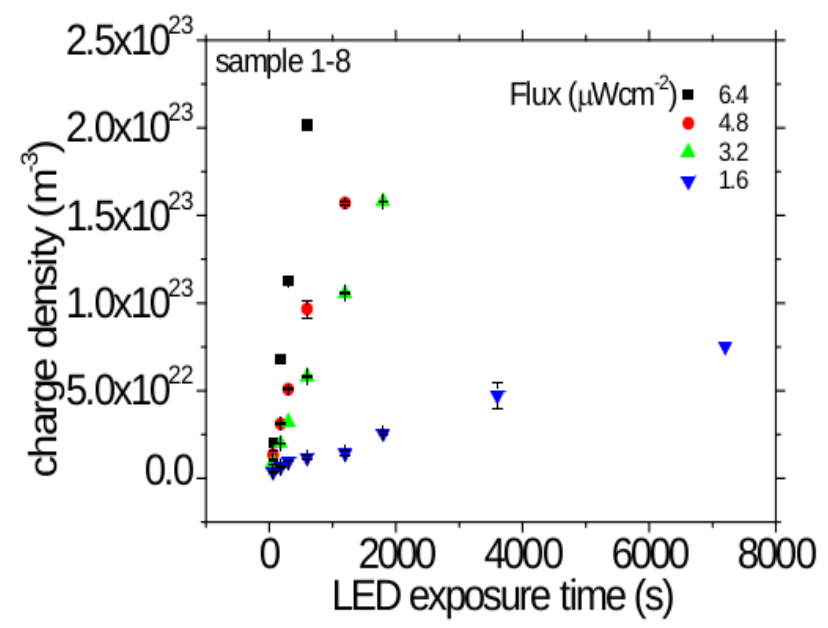

Figure 10: Charge build up in the device on exposure to a constant low light flux. In this mode the device is operating as an ultra low light level photodetector.

\section{Conclusion}

We have demonstrated an organic electronic device in which we observe carriers with a lifetime of over one hour. Carriers with lifetimes at room temperature in disordered organic semiconductors exceeding 0.3 seconds have not been previously been reported. Using a combination of experimentation and simulation, we attribute this long lifetime to band bending which drives electrons and holes apart, spatially separating them within the device shortly after photogeneration. The holes are then stored in a hole-rich electron-poor region of the device. We demonstrate that by 
carefully tuning the energetic landscape of the device carrier lifetimes can be significantly increased. This principle opens up the possibility of developing a new generation of plastic electronic ultra low light level photodetectors and memory elements.

\section{Acknowledgements}

This work was supported by 973 program (2014CB643506), the Natural Science Foundation of China (No. 21221063, 51373063), the Research Fund of Key Laboratory of Physics and Technology for Advanced Batteries, Ministry of Education (Grant no. 201206). YG acknowledges financial support through a Bernoulli Fellowship from the University of Groningen; thanks Prof. Yu Liu for helpful discussion on the analysis of mass spectroscopy, and thanks Yan Zhang and Lulin Yan for the synthesis of $\mathrm{SiO}_{2}$ sol-gel

\section{References}

1. J. C. Hummelen, B. W. Knight, F. LePeq, F. Wudl , and J. Yao , C. L. Wilkins, "Preparation and characterization of fulleroid and methanofullerene derivatives." J. Organ. Chem. Vol. 60, pp. 532-538, 1995

2. G. Yu, G. Srdanov, J. Wang, H. Wang, Y. Cao, and A. J Heeger, "Large area, full-color, digital image sensors made with semiconducting polymers”, Synth. Met., Vol. 111, pp. 133137,2000

3. M.M. Voigt, R.C.I. Mackenzie, S.P. King, C.P. Yau, P. Atienzar, J. Dane, P.E. Keivanidis, I. Zadrazil, D.C. Bradley, and J. Nelson, "Gravure printing inverted organic solar cells: The influence of ink properties on film quality and device performance ”, Sol. Energ. Mat. and Sol. Cells Vol. 105, pp. 77-85, 2012

4. J. Liang, L. Li, X. Niu, Z. Yu and Q. Pei, "Elastomeric polymer light-emitting devices and displays”,Nature Photonics, Vol. 7, pp. 817-824, 2013

5. M. Kaltenbrunner, M. S. White, E. D. Głowacki, T. Sekitani, T. Someya, N. S. Sariciftci and S. Bauer, "Ultrathin and lightweight organic solar cells with high flexibility",Nat. Commun. , Vol. 3, pp. 770, 2012

6. T. Han, Y. Lee, M. Choi, S. Woo, S. Bae, B. Hee Hong, J. Ahn and T. Lee, "Extremely efficient flexible organic light-emitting diodes with modified graphene anode", Nat. Photon., Vol. 6, pp. 105-110, 2012

7. C. Melzer, H. Seggern, "Organic electronics: Enlightened organic transistors",Nat. Mat.,Vol. 9, pp. 470-472, 2010

8. S. Pang, S. Yang, X. Feng, and K. Müllen, "Coplanar Asymmetrical Reduced Graphene Oxide-Titanium Electrodes for Polymer Photodetectors”, Adv. Mater. , Vol. 24, 1566-1570, 2012 
9. X. Wang, W. Song, B. Liu , G. Chen, D. Chen, C. Zhou, and G. Shen, "High-Performance Organic-Inorganic Hybrid Photodetectors Based on P3HT:CdSe Nanowire Heterojunctions on Rigid and Flexible Substrates”, Adv. Funct. Mater, Vol. 23, 1202-1209, 2013

10. X. Wang, O. Hofmann, R. Das, E. M. Barrett, A. J. de Mello, J. C. de Mello, and D. D. C. Bradley, "Integrated thin-film polymer/fullerene photodetectors for on-chip microfluidic chemiluminescence detection",Lab Chip, Vol. 7, 58-63, 2007

11. Z. Jin and J. Wang, "PIN architecture for ultrasensitive organic thin film photoconductors", Scientific Reports, Vol. 4, Article number: 5331, 2014

12. A. AY, Guilbert, J. M Frost, T. Agostinelliand, P. Ellis, S. Lilliu, J. E. McDonald, J. Nelson, "Influence of bridging atom and side chains on the structure and crystallinity of cyclopentadithiophene-benzothiadiazole polymers”, Chem. Mater., Vol. 26 2, pp 1226-1233, 2014

13. E. von Hauff, V. Dyakonov, and J. Parisi, "Study of field effect mobility in PCBM films and P3HT:PCBM blends”, Sol. Energ. Mat. and Sol. Cell., Vol. 87, pp. 149-156, 2005

14. Christopher M. Proctor, ,Chunki Kim,Dieter Neher,Thuc-Quyen Nguyen, "Nongeminate Recombination and Charge Transport Limitations in Diketopyrrolopyrrole-Based SolutionProcessed Small Molecule Solar Cells”, Adv. Fun. Mat.,Vol. 23, pp. 3584-3594, 2013

15. [1] R. A. Street, "Localized state distribution and its effect on recombination in organic solar cells", Phys. Rev. B, Vol. 84, 075208, 2011

16. Y. Kim, T. Kim,B. Kim, D. Lee,H. Kim, B. Ju, K. Kim, pp. 1566-1199, “Transient photovoltage and dark current analysis on enhanced open-circuit voltage of polymer solar cells with hole blocking TiO2 nanoparticle interfacial layer", Organ. Electron., Vol. 14, pp. 1749-1754, 2013

17. J. Lorrmann, B. H. Badada, O. Inganäs, V. Dyakonov and C. Deibel, "Charge carrier extraction by linearly increasing voltage: Analytic framework and ambipolar transients." $J$. Appl. Phys. Vol. 108, pp. 113705-113705, 2010

18. G. Garcia-Belmonte, P. P. Boix, J. Bisquert, M. Sessolo, H. J. Bolink, "Simultaneous determination of carrier lifetime and electron density-of-states in P3HT:PCBM organic solar cells under illumination by impedance spectroscopy", Sol. Energ. Mat. and Sol. Cell., Vol. 94, pp. 366-375, 2010

19. C. G. Shuttle, B. O’Regan, A. M. Ballantyne, J. Nelson, D. D. C. Bradley, J. de Mello, and J. R. Durrant, "Experimental determination of the rate law for charge carrier decay in a polythiophene: Fullerene solar cell”, Appl. Phys. Lett., Vol. 92, 093311, 2008

20. T. Kirchartz, B.E. Pieters, J. Kirkpatrick, U. Rau, J. Nelson, "Recombination via tail states in polythiophene: fullerene solar cells”, Phys. Rev. B, Vol. 83, 115209, 2011 
21. J. Cabanillas-Gonzalez, T. Virgili, A. Gambetta, L. Lüer, G. Lanzani, T. D. Anthopoulos, and D. M. de Leeuw, "Subpicosecond photoinduced Stark spectroscopy in fullerene-based devices", Phys. Rev. B Vol. 75, 045207, 2007

22. C. G. Shuttle, B. O’Regan, A. M. Ballantyne, J. Nelson, D. D. C. Bradley, J. de Mello, and J. R. Durrant, "Experimental determination of the rate law for charge carrier decay in a polythiophene: Fullerene solar cell”, Appl. Phys. Lett., Vol. 92, 093311, 2008

23. C. G. Shuttle, N. D. Treat, J. D. Douglas, J. M. J. Fréchet, M. L. Chabinyc, "Deep Energetic Trap States in Organic Photovoltaic Devices”, Adv. Energ. Mater, Vol. 2, pp.111-119, 2012

24. D. Taguchi, T. Shino, X. Chen, L. Zhang, J. Li, M. Weis, T. Manaka, and M. Iwamoto, “Analyzing carrier lifetime of double-layer organic solar cells by using optical electric-fieldinduced second-harmonic generation measurement”,Appl. Phys. Lett. Vol. 98, 133507, 2011

25. A. Foertig, A. Wagenpfahl, T. Gerbich, D. Cheyns, V. Dyakonov, and C. Deibel, "Nongeminate Recombination in Planar and Bulk Heterojunction Organic Solar Cells", Adv. Energy Mater., Vol. 2, pp. 1483-1489, 2012

26. B. Lüssem, M. Riede, and K. Leo,“Doping of organic semiconductors”, Phys. Status Solidi A 210, No. 1, 9-43, 2013

27. Wolfgang Brütting, Chihaya Adachi, "Physics of Organic Semiconductors",Section 4.3.2, "Dopants in organic semiconductors", Second Edition, John Wiley \& Sons, 2012

28. J. W. Ryan, T. Kirchartz, A. Viterisi, J. Nelson, and E. Palomares, "Understanding the Effect of Donor Layer Thickness and a MoO3 Hole Transport Layer on the Open-Circuit Voltage in Squaraine/C60 Bilayer Solar Cells”, J. Phys. Chem. C, Vol. 117, pp. 19866-19874, 2013

29. R. C. I. MacKenzie, T. Kirchartz , G. F. A. Dibb , and J. Nelson, "Modeling Nongeminate Recombination in P3HT:PCBM Solar Cells", J. Phys. Chem. C, Vol. 115 , pp 9806-9813, 2011

30. R. Hanfland, M.A. Fischer, W. Brütting, U. Würfel, R.C.I. MacKenzie , "The physical meaning of charge extraction by linearly increasing voltage transients from organic solar cells”, Appl. Phys. Lett. Vol. 103, 063904, 2013

31. T. Kirchartz, W. Gong, S. Hawks, T. Agostinelli, and Roderick C. I. MacKenzie, Y. Yang, J. Nelson, "Sensitivity of the Mott-Schottky Analysis in Organic Solar Cells", J. Phys. Chem. C, Vol. 116, pp 7672-7680, 2012

32. R. N. Hall, "Electron-hole recombination in germanium”, Phys. Rev., Vol. 87, p. 387, 1952

33. J. Nelson, Phys. Rev. B, "Diffusion-limited recombination in polymer-fullerene blends and its influence on photocurrent collection", Vol. 67, 155209, 2003

34. F. Deschler, D. Riedel, B. Ecker, E. Da Como, E. von Hauff, Roderick. C. I. MacKenzie, "Increasing organic solar cell efficiency with polymer interlayers", Phys. Chem. Chem. 
Phys., Vol. 15, 764-769, 2013

35. D. Amarasinghe Vithanage, A. Devižis, V. Abramavičius, Y. Infahsaeng, D. Abramavičius, R. C. I. MacKenzie, P. E. Keivanidis, A. Yartsev, D. Hertel, J. Nelson, V. Sundström and V. Gulbinas, "Visualizing charge separation in bulk heterojunction organic solar cells", Nature Communications, 4, Article number: 2334

36. R. C.I. MacKenzie, C. G Shuttle, M. L. Chabinyc, J. Nelson, "Extracting microscopic device parameters from transient photocurrent measurements of P3HT: PCBM solar cells", Adv. Energy Mat., Vol. 2, pp. 662-669, 2012 


\section{Supplementary information for: Engineering ultra long charge carrier lifetimes in plastic electronic devices at room temperature.}

Yajun Gao (高亚军), ${ }^{1,2, * *}$ Roderick.C.I. Mackenzie ${ }^{3, *, * *}$, Yang Liu (刘洋) ${ }^{1}$, Bin Xu (徐斌) ${ }^{1}$, Paul. H. M. van Loosdrecht, ${ }^{1,2,4, \$}$ and Wenjing Tian（田文晶） ${ }^{1, \#}$

${ }^{1}$ State Key Laboratory of Supramolecular Structures and Materials, Jilin University, 2699 Qianjin Street, Changchun 130012, China.

${ }^{2}$ Optical Condensed Matter Physics, Zernike Institute for Advanced Materials, University of Groningen, Nijenborgh 4, 9747AG Groningen, The Netherlands.

${ }^{3}$ Faculty of Engineering, The University of Nottingham, University Park, Nottingham, NG7 2RD, UK

${ }^{4}$ II. Physikalisches Institut, University of Cologne, Zülpicher Strasse 77, 50937 Cologne, Germany.

Corresponding authors:

*roderick.mackenzie@nottingham.ac.uk

\$pv1@ph2.uni-koeln.nl

\#wjtian@jlu.edu.cn

**Both authors contributed equally to this work.

\section{Full details of device fabrication:}

$\mathrm{SiO}_{2}$ sol-gel was synthesized by mixing $1 \mathrm{~mL}$ Tetraethylorthosilicate (TEOS) with $10 \mathrm{~mL}$ ethanol, and added with $0.4 \mathrm{~mL} \mathrm{HCl}(0.72 \mathrm{~mol} / \mathrm{L})$ in a drop-wise fashion. The solution was stirred for $2 \mathrm{~h}$ and kept for $24 \mathrm{~h}$ under the ambient condition. $\mathrm{A} \mathrm{SiO}_{2}$ layer $(70-170 \mathrm{~nm})$ was prepared by spin-coating the $\mathrm{SiO}_{2}$ sol-gel with a speed from 3500 to $800 \mathrm{rpm}$ on an ITOcoated glass substrate, followed with thermal annealing at $350 \mathrm{C}$ for $30 \mathrm{~min}$ in a glove box. NPB film (70-335 $\mathrm{nm}$ ) and $\mathrm{Al}$ (or $\mathrm{Cu}$ ) electrode $(30 \mathrm{~nm})$ were thermally deposited consecutively with a deposition speed less than $1 \AA / \mathrm{s}$ under a vacuum of $5 \times 10^{-4} \mathrm{~Pa}$. The longest lifetimes were achieved with170 nm thick layer of $\mathrm{SiO}_{2}$ and a $100 \mathrm{~nm}$ thick layer of NPB. The effective surface area for the device is $5 \mathrm{~mm}^{2}$. PS layer $(45-\mathrm{nm})$ on ITO-coated glass was prepared by spin-coating PS solution in toluene $(12.5 \mathrm{mg} / \mathrm{mL})$ with a speed of 3500 rpm, and PS layer $(19,36$, and $62 \mathrm{~nm})$ on $\mathrm{SiO}_{2}$ layer was prepared by spin-coating PS solution in toluene $(3.1,6.3$, and $12.5 \mathrm{mg} / \mathrm{mL}$ ) with a speed of $7000 \mathrm{rpm}$, followed with thermal 
annealing at $90 \mathrm{C}$ for $3 \mathrm{~h}$ in a glove box. TEOS was purchased from J\&K Scientific. NPB was purchased from Jilin Optical and Electronic Materials Co. Ltd. PS was purchased from Sigma Aldrich. All materials were used without further purification. The thickness was characterized with a Veeco DEKTAD 150 surface profilometer.

The CELIV set up consists of a pulsed laser (Continuum Minilete TM Nd:YAG, tripled to 355 $\mathrm{nm}$ ), a digital delay generator (Stanford Research System DG645), a function generator (Stanford Research System DS345), and an oscilloscope (Tektronix MSO 4054) for signal observation and recording. Photo-CELIV measurements are performed under ambient conditions, and samples are irradiated through the ITO side by one $10 \mathrm{~ns}, 355 \mathrm{~nm}$ laser flash, with the pulse energy flux adjustable up to $180 \mu \mathrm{J} / \mathrm{cm}^{2}$. In the experiments we typically used $110 \mu \mathrm{J} / \mathrm{cm}^{2}$

\section{Optical absorption spectrum of NPB}

Figure S1 shows the optical absorption spectrum of NPB used in the device model. The data was measured using a Shimazu UV-3100 spectrometer.

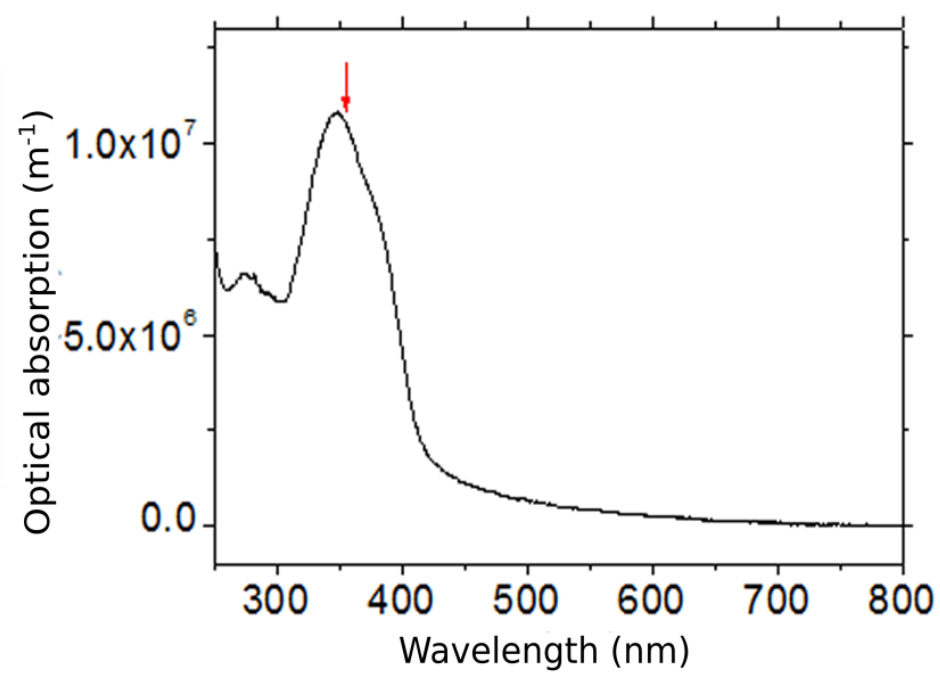

Figure S1: The optical absorption spectra of the NPB film as measured by UV-Vis. The red arrow indicates the wavelength used for excitation in the experiments.

\section{Current transients as a function of bias voltage (Vstart)}

Figure S2a and S2b show experimental and simulated current transients as a function of Vstart respectively. It can be seen that as Vstart is increased, in both the experimental data and the simulations, the position of the current maxima shifts to earlier times.

a.

b. 

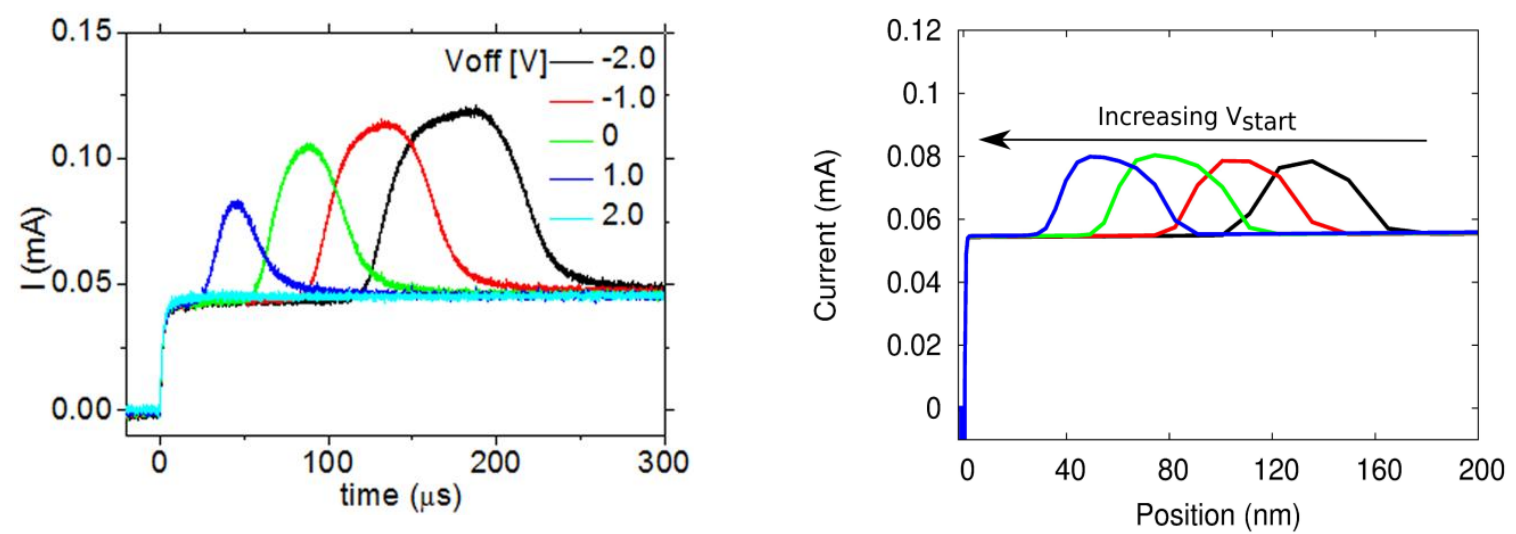

Figure S2: The influence of the initial voltage on the experimental transient (left). The influence of the initial voltage on the simulated transient (right).

\section{The influence of the conducting contact material upon the transient.}

Figure S3 shows the influence of changing the conducting contact from Aluminum to Copper. It can be seen that when copper contacts are used the charge carriers still remain in the device a long time. However the shape of the extracted transient becomes more spread out as a function of time. This suggests that the copper contact is changing the energetic profile within the device but not enough to reduce the recombination rate.
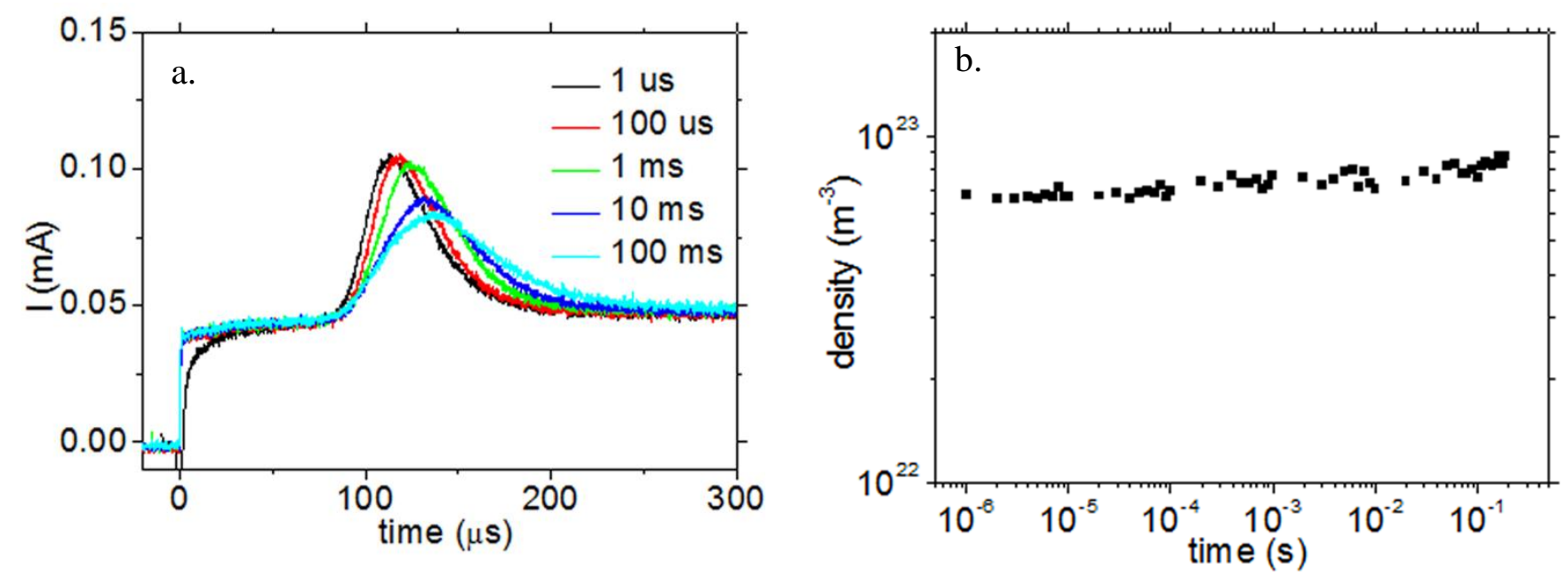

Figure S3: When the Al contact is replaced by a copper contact it can be seen that the pulse spreads out more as a function of time. Long lifetimes are still observed with the Cu contact.

\section{Simulation parameters}




\begin{tabular}{lll}
\hline Parameter & Value & Units \\
\hline Effective density of states & $3.0 \times 10^{23}$ & $\mathrm{~m}^{-3}$ \\
Density of electron/hole trap states & $1.0 \times 10^{10}$ & $\mathrm{~m}^{-3} \mathrm{eV}^{-1}$ \\
Free electron-trapped electron capture cross section & $1.0 \times 10^{-20}$ & $\mathrm{~m}^{-2}$ \\
Trapped electron-free hole capture cross section & $1.0 \times 10^{-20}$ & $\mathrm{~m}^{-2}$ \\
Free electron-trapped hole capture cross section & $1.0 \times 10^{-20}$ & $\mathrm{~m}^{-2}$ \\
Free hole-trapped hole capture cross section & $1.0 \times 10^{-20}$ & $\mathrm{~m}^{-2}$ \\
Density of carriers on the majority p-contact & $1.0 \times 10^{10}$ & $\mathrm{~m}^{-3}$ \\
Density of carriers on the majority n-contact & $1.0 \times 10^{10}$ & $\mathrm{~m}^{-3}$ \\
Electron tail slope & 40 & $\mathrm{meV}$ \\
Hole tail slope & 40 & $\mathrm{meV}$ \\
\hline
\end{tabular}

Table S1: Simulation parameters used in the model.

The main result presented in the paper is that if the material is doped, the bands bend upwards creating a hole rich and electron poor region, because of the low electron concentration under the potential hill, holes live for a long time. This is a general result and is observed irrespective of the exact values of the simulation parameters chosen. Furthermore, it is important to note that within the manuscript we do not state that the chosen model parameters correspond exactly to those in the real device nor that they are the only set of material parameters which could reproduce the experimental results. It should also be noted that fugue $8 \mathrm{~b}$ is a prediction not a fit, further giving validity to our model.

\section{Quality of the $\mathrm{SiO}_{2}$ layer}

$\mathrm{SiO} 2$ layers deposited from sol-gel are known not to be as effective insulators as $\mathrm{SiO}_{2}$ layers deposited by evaporation. In order to test the quality of our layer an ITO/SiO $2 / A l$ device was fabricated and current voltage curves measured (figure S4). It can be seen that when voltages of over 1-2 V are applied to the device current can be seen to flow. This suggests that impurities have been left in the $\mathrm{SiO}_{2}$ from the fabrication process, and it can only provide 1-2 eV of electrical isolation. Therefore in the model we approximate the $\mathrm{SiO}_{2} / \mathrm{NPB}$ interface with a $2 \mathrm{eV}$ step. We should however highlight that because the mobility of the $\mathrm{SiO}_{2}$ layer is low, introducing a step (of any magnitude) in the band structure does not significantly influence the main conclusions drawn from the simulations. 


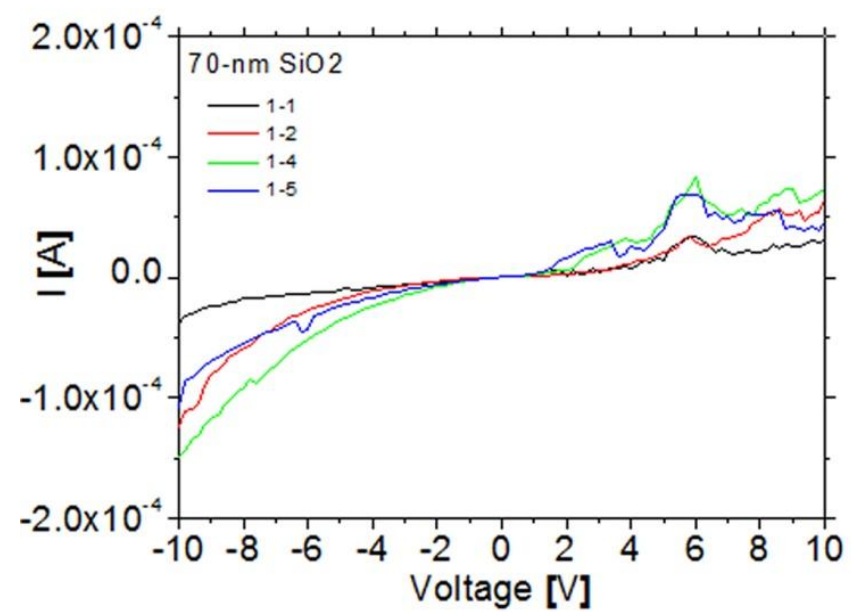

Figure S4: Conduction of current through defects in a layer of $\mathrm{SiO}_{2}$, deposited from sol-gel. 


\section{The identity of the dopants}

In order to determine the source of the doping, we performed optical mass spectroscopy on as received NPB, the result is shown in figure S5.

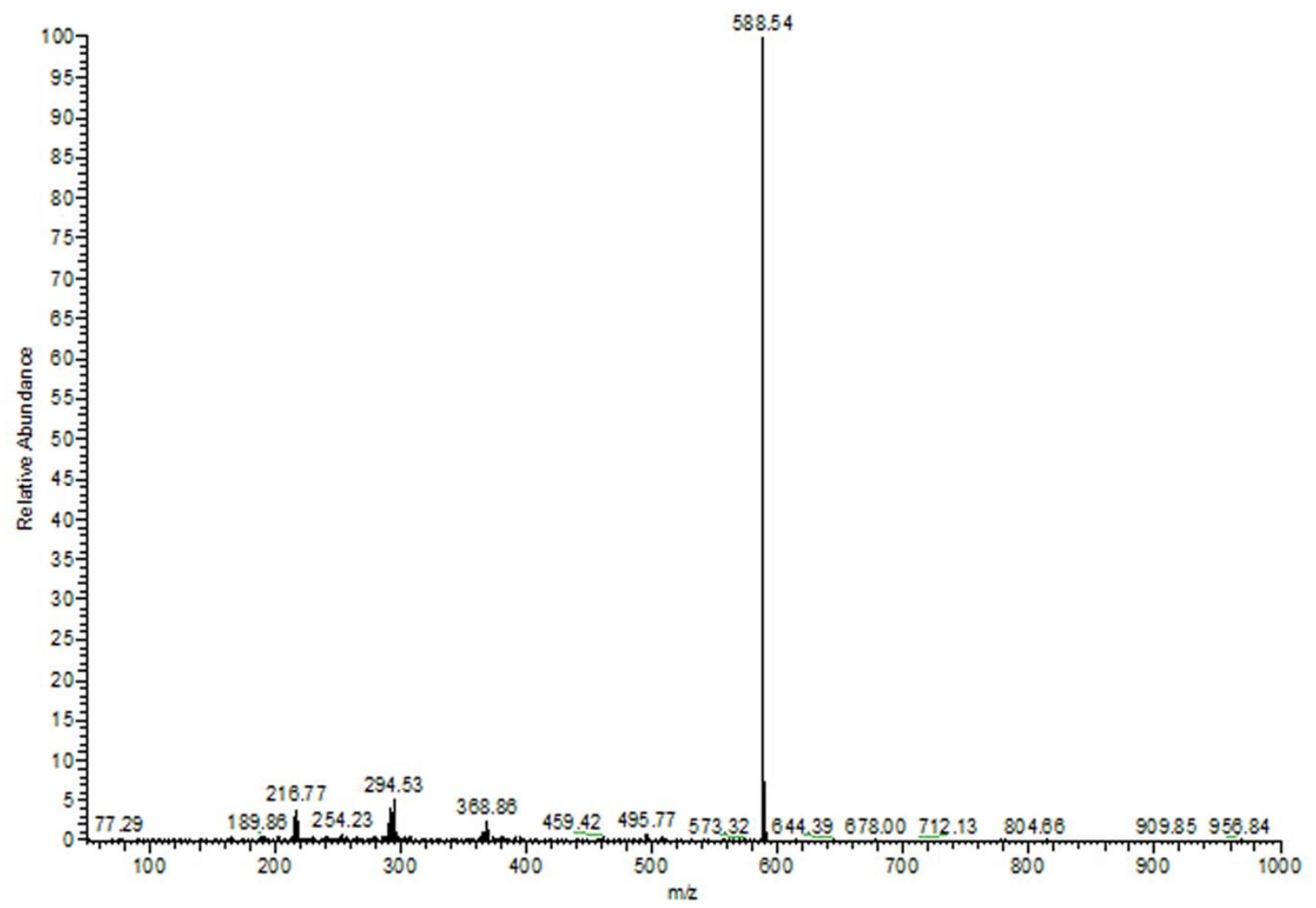

Figure S5: Mass spectrum of NPB, the large peak at $M W=588$ represents NPB. Other smaller peaks at 368.86, 294.53, 216.77 , and 189.86 can also be seen.

The large peak dominating the spectrum at $588.54 \mathrm{~m} / \mathrm{z}$ represents pure NPB. There are also smaller but significant peaks at $368.86,294.53,216.77$ and 189.86 . These peaks a can be attributed to three bi-products formed during the synthesis of NPB which are depicted in figure S6. 

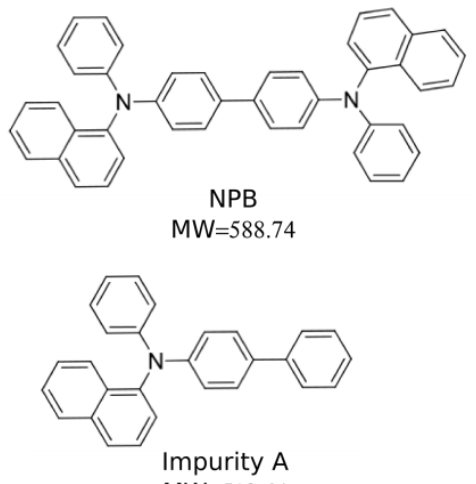
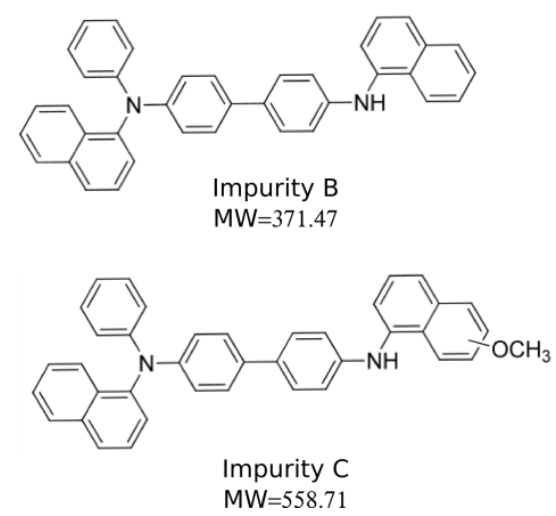

Figure S6: Possible impurities found in NPB the molecular weight $(M W)$ of each molecule is also shown.

The peaks observed at 371.47 and at 189.25 in figure S5 are very probably due to fragments of impurity $\mathrm{C}$ (see figure S7 top). A further contribution to the $371.47 \mathrm{~m} / \mathrm{z}$ peak, and the cause of the $216.77 \mathrm{~m} / \mathrm{z}$ peak could be due to NPB breaking up in the mass spectrometer (see figure S7 bottom). For the peak located at 294.53, there are two possible origins, NPB (figure S8 top) or impurity A (figure S8 bottom). The peak at 216.77 could have contributions from both NPB (figure S9 top) and impurity B (figure S9 bottom). Thus from the mass spectrum of our NPB sample we can say that impurity $\mathrm{C}$ is almost certainly present, while impurities $\mathrm{B}$ and $\mathrm{C}$ could also be present. The source of the dopants is discussed below.
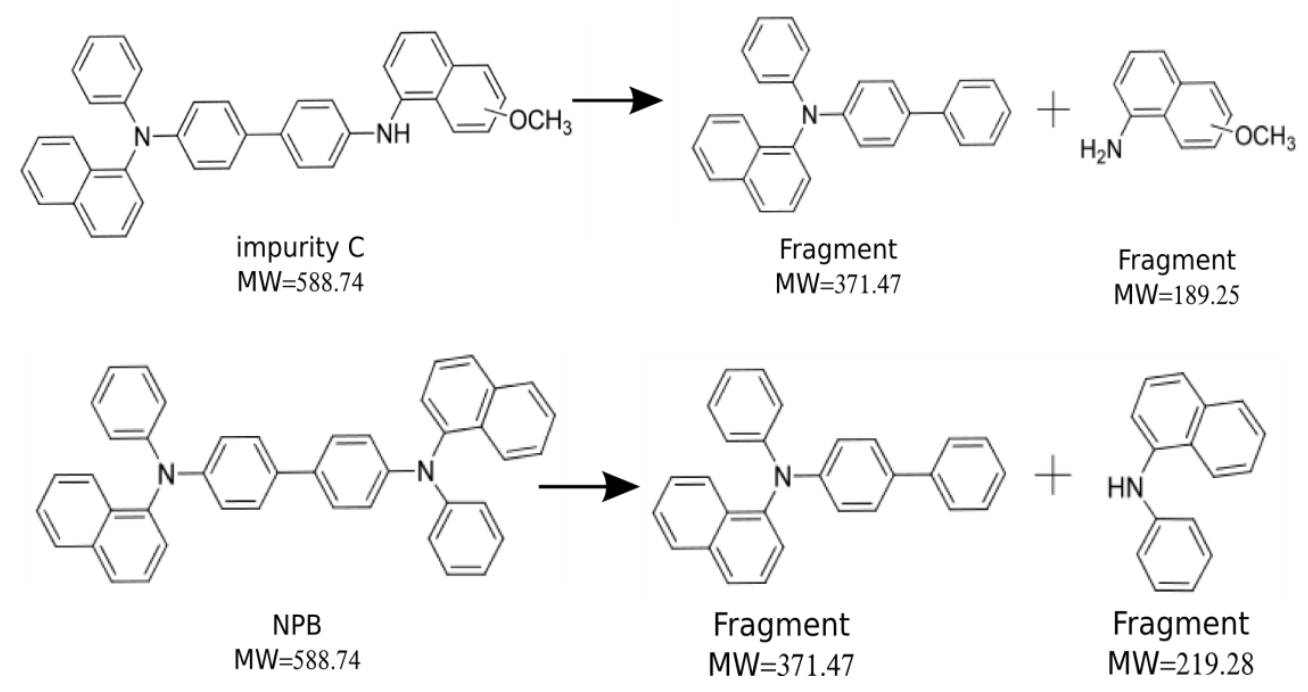

Figure S7: Degradation of impurity C into two compounds with molecular weight of $371.47 \mathrm{~m} / \mathrm{z}$ and $189.25 \mathrm{~m} / \mathrm{z}$. A fragment of pure NPB could also contribute to the peak at $371.47 \mathrm{~m} / \mathrm{z}$. 


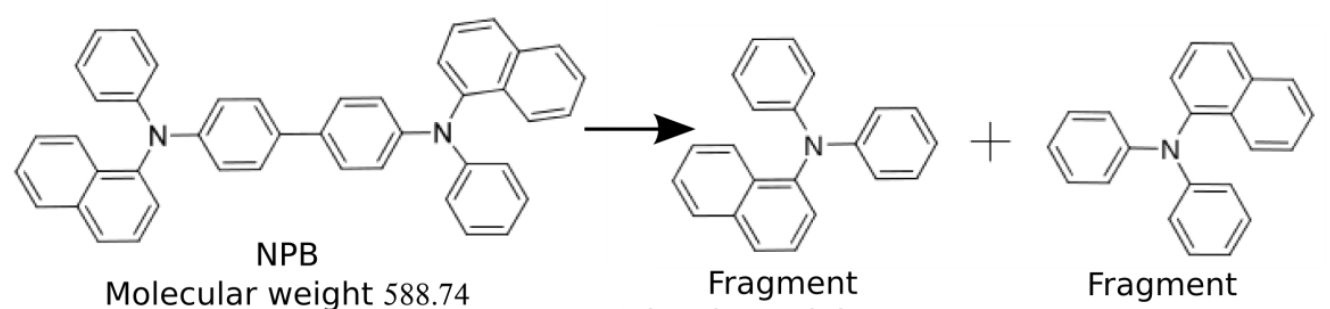

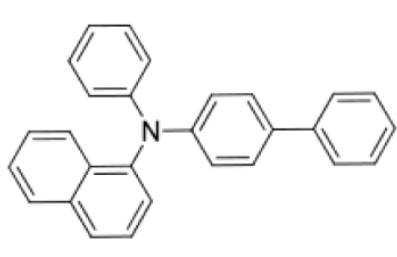

Impurity A

Molecular weight 371.47

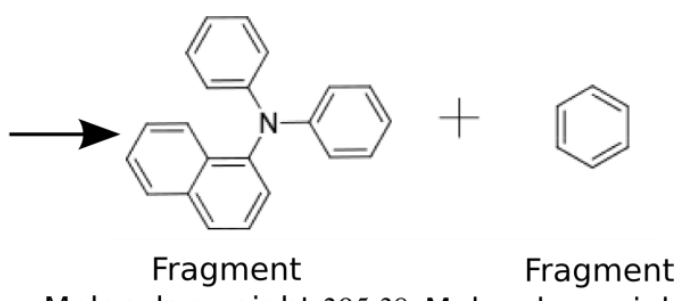

Molecular weight 295.38 Molecular weight 78.11

Figure S8: Possible causes of the peaks in molecular weight seen at $294.53 \mathrm{~m} / \mathrm{z}$.
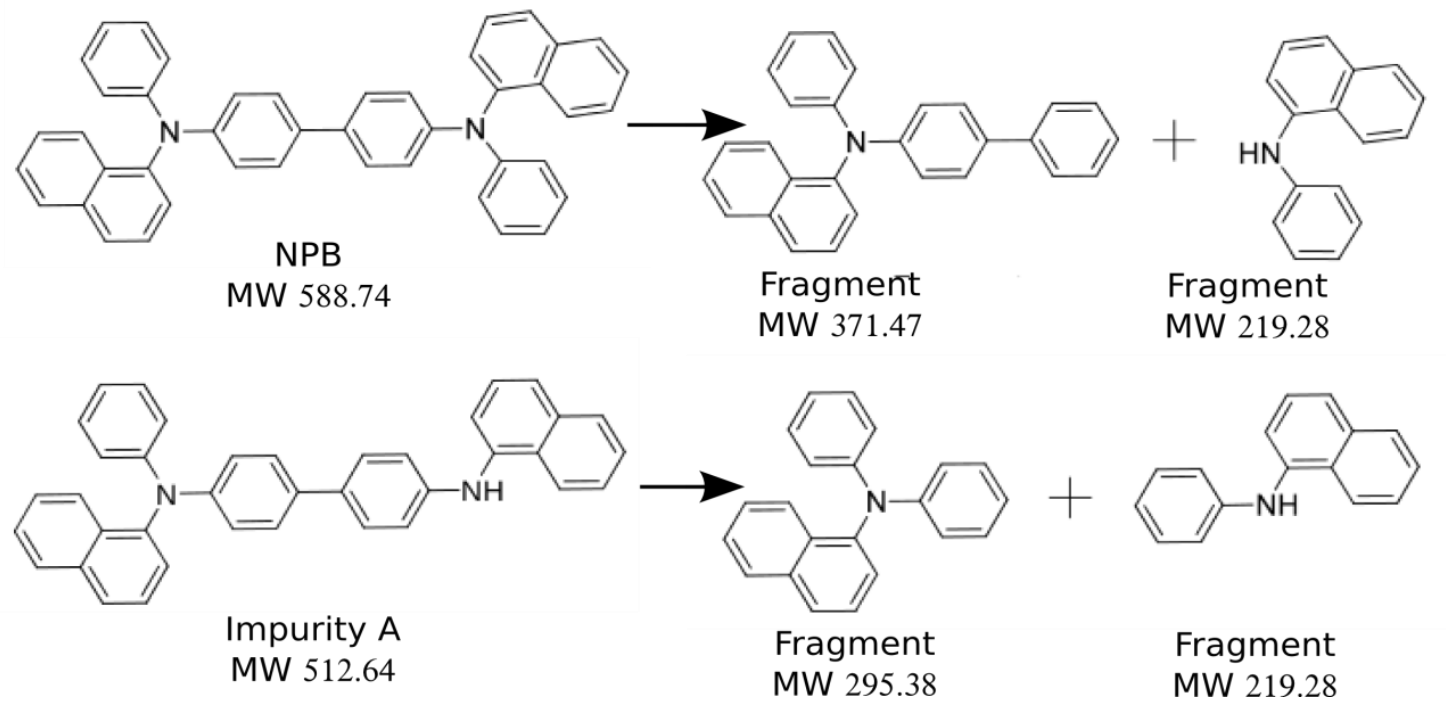

Figure S9: Possible causes for the peak at $216.77 \mathrm{~m} / \mathrm{z}$

\section{The source of the dopants}

The formation of these bi-products can be traced back to the synthesis of NPB. NPB is synthesized by the arylation of 4,4-Diiodobiphenyl (1) with N-Phenyl-1-naphthylamine (2), this is depicted in figure $\mathrm{S} 10$ and described in references [S1,S2]: 


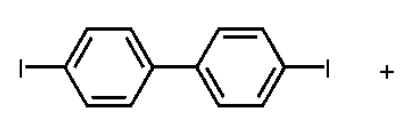

(1)

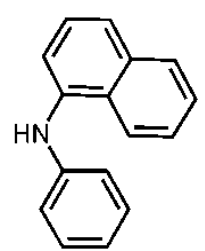

(2)
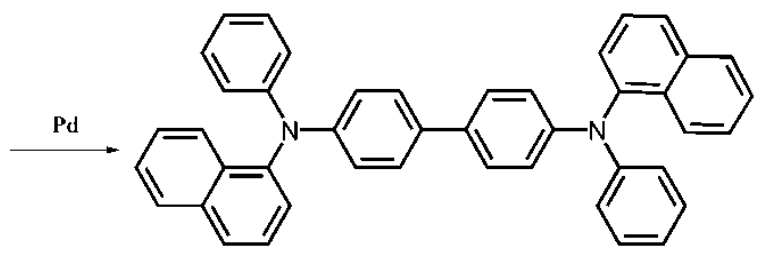

(NPI)

Figure S10: The production of NPB from 4,4-Diiodobiphenyl (1) and N-Phenyl-1-naphthylamine

The 4,4-Diiodobiphenyl for this reaction was produced by reacting biphenyl with Iodine in Nitrogen dioxide, see figure S11 [S3]

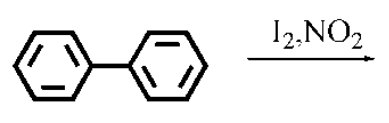

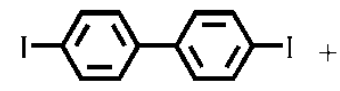

(1)

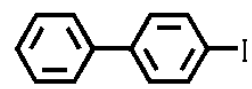

(3)

Figure S11: The production of 4,4-Diiodobiphenyl (1) with the formation of 4-iodobiphenyl (2)as a bi-product

This reaction produced a small amount of 4-iodobiphenyl (3 in figure S11), which contaminated reactant 1 in figure S10. Impurity A was then formed through the reaction of 4-iodobiphenyl with N-Phenyl-1-naphthylamine, this is depicted in figure S12.

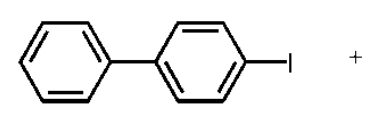

(3)

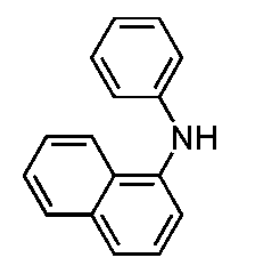

(2)

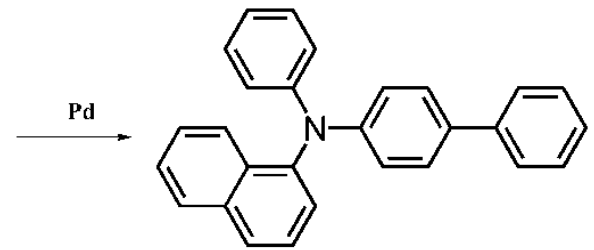

Figure S12: Formation of impurity A.

The N-Phenyl-1-naphthylamine (reactant 2) in figure S10 was synthesized as shown in figure S13.

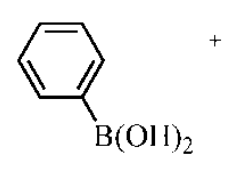<smiles>Nc1cccc2ccccc12</smiles>

(4)

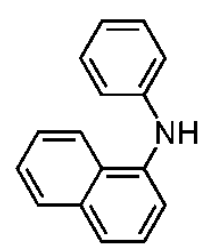

(2)

Figure S13: Formation of N-Phenyl-1-naphthylamine [S4].

Some naphthylamine (4 in figure S13) remains unreacted, and can react to produce impurity B and $\mathrm{C}$ without the methoxy group, this is show in figure S14. 


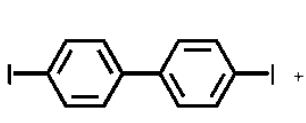

(1)<smiles>Nc1cccc2ccccc12</smiles>

(4)
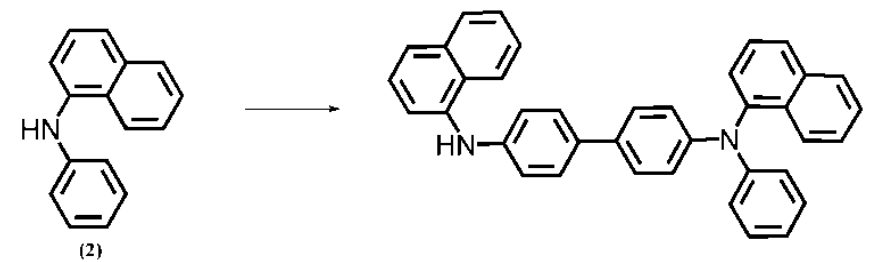

Figure S14: The formation of impurity B and C without the methoxy group.

\section{Mott-Schotky data}

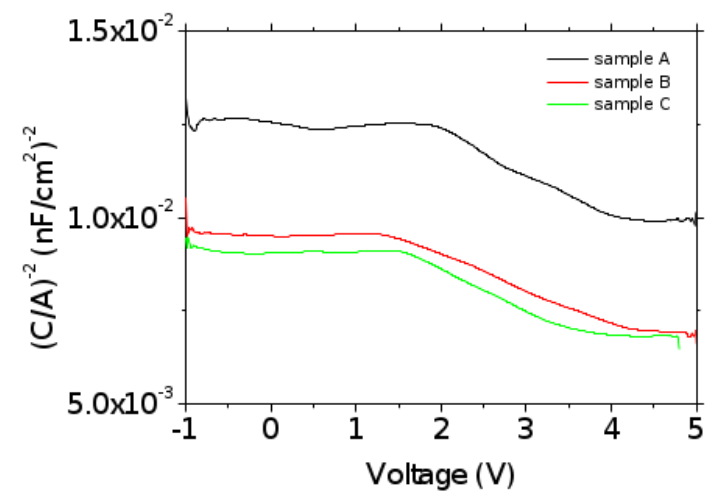

Figure S15: Mott-Schotky plots of three ITO/SiO2/NPB/Al devices.

\section{Life time data for the Glass/ITO/PMMA/NPB/Al structure}

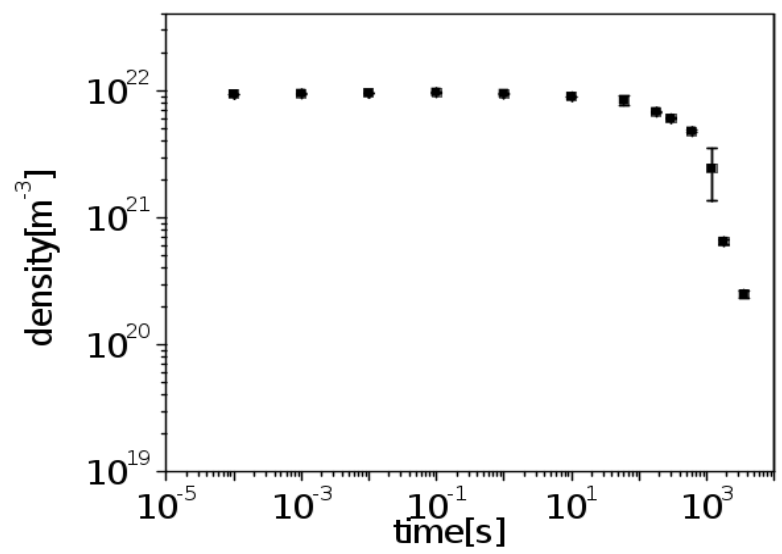

Figure S16: The integrated photocharge extracted from a Glass/ITO/PMMA/NPB/Al structure as a function of $t_{\text {delay. }}$.

\section{Current features after $400 \mu \mathrm{s}$}

The current transient from our device consists of two components; a) The hill of photogenerated charge extracted from the active region centered around $\sim 100 \mu \mathrm{s}$; and $\mathrm{b}$ ) the a square shaped box of 
charge spanning $0 \mu \mathrm{s}$ to $400 \mu \mathrm{s}$, which is due to geometric capacitive charge being removed from the contacts by the voltage ramp. At time $400 \mu \mathrm{s}$, all the photogenerated charge has been removed from the device, and the experiment is effectively over. We therefore simply return the power supply from $\mathrm{V}_{\max }(\sim 10 \mathrm{~V})$ to $0 \mathrm{~V}$, at which point the geometric capacitive charge which has been removed from the contacts must flow back into the device to bring it to equilibrium - this is the cause for the negative current spike at $400 \mu \mathrm{s}$.

After $400 \mu$ s, we apply a second voltage ramp to check that all the photogenerated charge has been removed. This second voltage ramp invariably only extracts geometric capacitive charge from the contacts of the device and no photocurrent, hence the current transient after $410 \mu$ s is flat.

\section{Removal of electrons from the device}

In the main body of the text we describe, photogenerated electrons being swept out of the device first by the curved band structure leaving only holes within the device. This can be seen in figure 3 where a large negative spike in current can be seen upon photoexcitation at time $-100 \mu$ s.

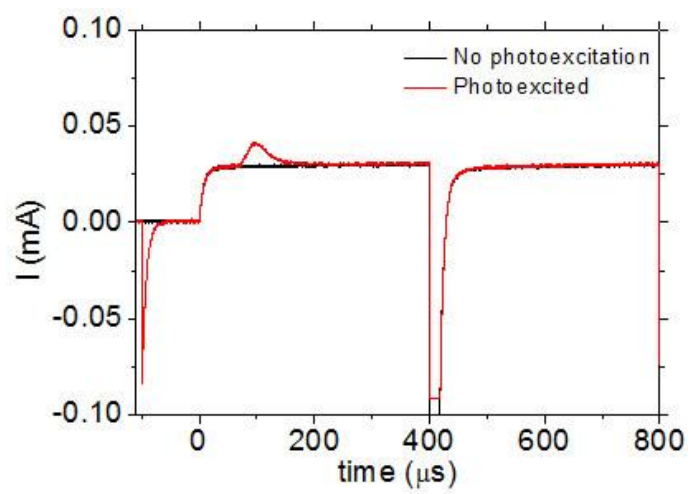

Figure S17: The current extracted from the device after photoexcitation (red) and with no photo excitation.

[S1] European Patent Application EP 1018505A2

[S2] J. H. Cho, Y. Ryu, S. H. Oh, J. K. Kwon, E. K. Yum, Bull. Korean Chem. Soc., Vol. 32, p. 2461,2011

[S3] Y. Noda and M. Kashima, Tetrahedron Lett. Vol. 38, pp. 6225-6228,1997

[S4] M. Nasrollahzadeh a, A. Azarian, A. Ehsani, A. Zahraei, Tetrahedron Lett. Vol. 55, pp. 28132817,2014 